\title{
La excelencia de las transformaciones urbanas a través del reciclaje
}

\author{
Carlos Hernández Pezzi *
}

\section{Resumen}

Los puntos de partida del reciclaje de barrios cambian constantemente. El ámbito del reciclaje ha pasado de ser esporádico o a-sistémico a convertirse en una herramienta conceptual y técnica para la calidad de vida; eso sí, partiendo del escenario real de recesión económica y de la inflexión en las políticas urbanas producida a partir de la crisis. Se trata de poner al día la teoría y la práctica a través de las fórmulas y las experiencias transversales más recientes de prácticas en los distritos, desplegando actuaciones espaciales sobre la ciudad de las personas, mediante la innovación social, con la energía de una nueva gobernanza. En el contexto de la innovación de las ciudades españolas, la arquitectura cambia de visión si se trata como un factor de regeneración y revitalización a distintas escalas. Se trata de definir los retos y dimensiones del reciclaje urbano en un momento crucial desde el discurso hacia el urbanismo más sostenible buscando la excelencia en los modelos de transformación de las ciudades, sus comunidades y sus entornos de desarrollo de la ciudadanía.

\section{Palabras clave}

Reciclaje; Excelencia; Barrios; Innovación Social; Medio Ambiente

\section{Abstract: The excellence of the urban transformations through recycling}

The starting points for the recycling of neighborhoods are constantly changing. The recycling sector has gone from being sporadic or a-systemic to become a tool for conceptual and technical quality of life yes, based on the real scenario of economic recession and the inflection in urban policy produced from the crisis. This is to update the theory and practice through the formulas and experiences transverse latest practices in the districts, deploying space activities over the city of people through social innovation, with the energy of new governance. In the context of innovation of Spanish cities, architecture, vision changes, if treated as a factor in regeneration and revitalization at different scales. It is about defining the challenges and dimensions of urban recycling at a crucial time since the speech to more sustainable urban development seeking excellence in transformation models of cities, their communities and their environments development of citizenship.

\section{Key words}

Recycling; Excellence; Neighborhoods; Social Innovation; Environment 


\section{Los retos culturales, sociales, ambientales y económicos del reciclaje urbano}

Con los antecedentes de la crisis inmobiliaria (Estefanía, 2011) que comenzó a entreverse en el $2^{\circ}$ semestre de 2006 y la aparición del "Manifiesto por una nueva cultura del territorio" que habían avisado de los efectos económicos y de la indigencia teórica del urbanismo asumido y practicado en España, las políticas urbanas sufrieron un punto de inflexión en 2010, cuando se empezó a comprender que la crisis no era coyuntural o cíclica, sino estanca y severa con muchos frentes a la vez. En ese año se celebró en Madrid un hito importante, la Sustainable Building Conference (SB10MAD), Edificación Sostenible Revitalización y Rehabilitación de barrios. El congreso se articuló en cuatro áreas temáticas con sus respectivos objetivos: A. Rehabilitación sostenible de barrios. Buenas prácticas: Intervenciones de rehabilitación a escala de barrio y con objetivos de sostenibilidad desde la experiencia de los distintos agentes implicados. B. Las técnicas tradicionales y la rehabilitación de barrios: El Patrimonio no sólo como legado material, sino como conocimiento integrado, expresado en técnicas, procesos y modelos de gestión de los recursos, con importantes lecciones para la sostenibilidad. C. Metodologías y herramientas de medida de la sostenibilidad: Búsqueda de aportaciones para la valoración de la eficiencia energética de la edificación rehabilitada, a escala de barrio. D. Ciudad, edificación y turismo: un reto mediterráneo: Considerar la incidencia de la actividad turística en la rehabilitación sostenible de los barrios de la ciudad mediterránea, entendida como riesgo y oportunidad.. VII Concurso Internacional de Buenas Prácticas para Mejorar las Condiciones de Vida organizado por el Programa de Naciones Unidas para los Asentamientos Humanos (UN-Habitat).
Este congreso vendría a levantar acta del estado de la cuestión de la sostenibilidad edificatoria en España y, meses después, otro hito contundente fue la desaparición el Ministerio de Vivienda, pasando a integrarse en el de Fomento como Secretaría de Estado. Estos dos acontecimientos coincidieron en señalar un momento clave en el conocimiento y la acción, que desvanecieron las esperanzas puestas en la posibilidad de planes integrales de rehabilitación energética y cambios en el modelo del sector de la construcción en España que se habían difundido en 2009 como vía para hacer frente a la explosión oficial de la burbuja inmobiliaria con la caída de Lehmann Brothers en septiembre de 2008.

Es de destacar el cambio producido en muy poco tiempo. El Informe de la Fundación IDEAS sobre el nuevo modelo productivo ha quedado superado por los acontecimientos y el cambio de estrategia económica conservadora del nuevo Gobierno 2011. Según la Fundación IDEAS, los cambios entre 2009 y 2025 en la composición del VAB y el empleo, son casi inversos a los actuales. Hemos de pasar del $34 \%$ al $45 \%$ en los Sectores renovados en 2025 y reducir del $66 \%$ al $55 \%$ el peso del resto hasta 2025. Casi el mismo porcentaje debe ser el de la composición del empleo en 2025, ya que debería ir del $36 \%$ al $47 \%$ en los Sectores renovados y nuevos sectores y bajar del $64 \%$ al $53 \%$ en el resto. En ambos casos estamos en un cambio cercano al $11 \%$ a favor del cambio.

Los Sectores renovados están identificados en los tres grandes actuales de Construcción, Turismo y Transporte que constituyen en el Escenario de Renovación Parcial en 2020 el 37,31\%. Los Nuevos sectores, Energías Renovables, Ecoindustrias, TIC, Biotecnología, Industria Aeroespacial, Industrias Culturales y Servicios Sociales serían el restante $62,68 \%$ en 2020 . Si hablamos del NMP, en 2025 serán del $45,72 \%$ y del $54,27 \%$ respectivamente. En 2025 los porcentajes del 
Escenario de renovación parcial serían del $33,82 \%$ y en el NMP del $41,87 \%$ respectivamente, lo que daría a las tendencias deseables en nuevos sectores, porcentajes del $66,18 \%$ y del $58,12 \%$. Es decir, estaríamos ante un cambio de una envergadura sin precedentes aunque no se cumplieran todos los pronósticos. Según este informe el Nuevo modelo Productivo debería ofrecer un nuevo marco de pesos relativos en los sectores renovados de Construcción, turismo $Y$ transporte para el año 2025, pero las expectativas razonables han saltado por los aires y la posibilidad de un horizonte no deseable en la transformación económica augura malos tiempos para la transformación de la construcción hacia un sector renovado en la vía de la sostenibilidad.

En 2012, la crisis ha devenido en crash multiorgánico y una profunda recesión se ha instalado en Europa, en tanto las políticas públicas han emprendido un camino incierto en el que prácticamente todo empieza a quebrarse, desde la confianza hasta las expectativas de un futuro mejor.

Si teníamos esbozado un discurso ahora tenemos dibujados numerosos interrogantes. ¿Qué elementos siguen siendo válidos en el escenario de recesión? ¿Qué cuestiones han de replantearse para re-evaluar las estrategias en la revitalización y la rehabilitación de barriadas? ¿Estamos ante una nueva reorientación de los modelos económicos vigentes en España? o ¿volveremos a la práctica anterior en cuanto se den las condiciones de mejora? Todas estas cuestiones obligan a revisar los retos culturales, sociales, ambientales y económicos del reciclaje urbano bajo la luz de una nueva perspectiva en la que los elementos de incertidumbre se insertan en un discurso todavía incipiente.

Desde el punto de vista del objetivo de la ciudad compacta no existen dudas acerca de la capacidad regeneradora de los barrios para configurar la nueva ciudad que emerge dentro de la existente. Es lo más eficiente, sostenible y equitativo para asentar la población en su entorno y para centrar en un ámbito espacial el conjunto de las acciones que más afectan a la eficiencia energética y la cohesión social en un mismo programa que, al reciclar, obtiene los mayores equilibrios de los recursos puestos en juego.

La cuestión ahora no es sólo cultural: se ha puesto completamente en crisis el modelo de la edificación nueva, de desarrollo masivo de nueva planta, de edificación extensiva con grandes ocupaciones de suelo, pero también el del mismo sistema que ha llevado de la hipoteca al desahucio en un solo período a centenares de miles de familias hipotecadas $y$ ha dejado sin vivienda a los que habían puesto su vida al servicio de un bien que se consideró un derecho y no se garantizó como tal.

Por la vía hipotecaria, la expropiación de la clase media ha sido letal para la emancipación de miles de jóvenes que están soportando la carga de una recesión sin precedentes, antes de poder reorientar el modelo económico tal y como se venía reclamando. El cambio de cultura territorial se ha hecho por la vía más traumática posible: Se ha paralizado todo el sector financiero, crediticio y constructivo de la construcción y no hay alternativa posible, porque no existe iniciativa viable, incluida la rehabilitación.

\section{Las dimensiones del reciclaje de barrios (tejidos, capas, itinerarios, entornos, nodos y barreras)}

La deuda pública de las familias, empresas, administraciones locales y estado, por este orden y los recortes de casi 40.000 millones de euros del Presupuesto español no auguran un cambio de tendencia en el corto o medio plazo, en tanto las políticas ambientales se encuentran en franca regresión, -véase el caso del litoral, las energías renovables, la cuestión de la energía nuclear y las 
demandas eléctricas-, de forma que estamos ante una situación de riesgo que ni siquiera en 2010 podíamos vislumbrar. La nueva formulación de las estrategias y las dimensiones del reciclaje se debe a que paradójicamente asistimos a una etapa dura para las estrategias blandas.

$\mathrm{Si}$ en los últimos tiempos se han puesto en marcha programas que van desde la "smart city" de la Misericordia en Málaga (Ministerio de Innovación y Ciencia, Junta de Andalucía y Ayuntamiento) y se ha reconocido con una mención de la BEAU XI las experiencias del proyecto "Esto no es un solar" de los arquitectos Di Monte y Grávalos con el Ayuntamiento de Zaragoza, también han surgido iniciativas como "Disueña tu barrio" en Zaragoza o "El Campo de la Cebada" en la Plaza de la Cebada de Madrid. Es decir, las estrategias pasan de la formulación de las TIC, de las soluciones urbanas "formales" a las "informales", del hardware al software en el planteamiento y de la mayor o menor incorporación de actores sociales a las iniciativas (Boned Purkiss, 2011).

En arquitectura la BEAU XI ha reconocido la Biblioteca Font de La Mina de Soldevilla arquitectos, como en la BEAU $X$ se reconoció el Plan de transformación del barrio de La Mina. Las dimensiones pueden ser concurrentes, pero el proceso de interacción no es unívoco, del hito a la trama existe una gama de intervenciones prácticamente interminable, que pueden tener más peso o menos en la transformación del entorno, pero que no son excluyentes, como ocurre con el Teatro Valle Inclán de Lavapiés de Paredes y Pedrosa, también reconocido con el Premio de la Arquitectura Española.

En tanto la cuestión del reciclaje de barrios se ha ido convirtiendo en un problema multi-dimensional con cada vez más ramificaciones, las herramientas del cambio siguen en manos de los mismos mecanismos de gestión administrativa del urba- nismo. Desde los campos de la acción pública se están operando con los instrumentos del último período. Agencias e institutos trabajan -a pesar de los severos recortes- en programas que parecen tener muchas incertidumbres sobre su viabilidad, a tenor del paro en seco de las políticas de vivienda pública, la caída de la rehabilitación y la previsible contrarreforma de la legislación de suelo y valoraciones refundida en 2008.

Conviene tener a mano la definición de reciclaje "reciclar. 1. tr. Someter un material usado a un proceso para que se pueda volver a utilizar. 2. tr. Dar formación complementaria a profesionales o técnicos para que amplíen y pongan al día sus conocimientos. 3. tr. Dar una nueva formación a profesionales o técnicos para que actúen en otra especialidad. 4. tr. Tecnol. Someter repetidamente una materia a un mismo ciclo, para ampliar o incrementar los efectos de este" (Drae, 2001) para no perder de vista las connotaciones a las que hacer frente en su traducción a la ciudad. EI componente material de las traducciones 0 interpretaciones a la escala espacial puede sufrir variaciones ilimitadas y dar lugar a malentendidos en su aplicación en los límites de la arquitectura con el urbanismo y la ordenación espacial. Las metodologías paramétricas del urbanismo tradicional son tan antiguas que contamos con casos excepcionales como el caso de los Indicadores Urbanos de Sostenibilidad del PGOU de Sevilla y otros, por ejemplo de la Agencia de Ecología Urbana de Barcelona, a los efectos de uso de metodologías de buenas prácticas, hoy por hoy, no generalizadas. $Y$ esto implica la manera de medir y ser medidos los elementos que conforman las dimensiones de las tareas abiertas a la intervención rehabilitadora.

Una primera cuestión alerta sobre la variedad de topologías de los tejidos, edades, características morfológicas y tipológicas. Una segunda cuestión muy importante es la de las capas desde el 
momento en que se estudian, no solo aquellas que están sobre rasante, sino las que están bajo rasante, redes de servicios e infraestructuras incluidas. La constitución de itinerarios y la enfatización de nodos -a veces no necesariamente hitos arquitectónicos, sino espaciales, estaciones de metro o paradas de autobús, espacios residuales o solares vacíos)- son cuestiones cada vez más importantes, ya que, contar solo con los barrios como unidades residenciales deja fuera cuestiones que se han resuelto desde la visión de la movilidad u otras.

Un caso paradigmático es el de los carriles-bici que unen tejidos separados y eliminan barreras antes invisibles, pero excluyentes de tránsitos peatonales con difícil continuidad. El carril bici integra barrios y mueve fronteras. Es un hecho que vincula las fórmulas de reequilibrio de un barrio con otro mucho más de lo que puede aparecer en una primera lectura. En Sevilla y Málaga, por estrategias distintas y con distinto acierto, se han integrado zonas a veces contradictoriamente aisladas por la movilidad automóvil, áspera y excluyente donde predomina sobre las demás.

La humildad de soluciones simplemente sensatas aminora la pulsión del desgaste de recursos (incluidos los excesos innecesarios de lenguaje), la peatonalización o la calma del tráfico son complementos esenciales en el plano referencial. El carril-bici induce a la geo-referencia de otros ámbitos y a la porosidad blanda entre barrios que, por sí sola, ya es un elemento de regeneración de primer orden (Puig, 2009).

\section{Los espacios. Convivencias: Modos y redes}

La escala del barrio las de distrito y las de ciudad suelen concebirse desde la arquitectura y la ingeniería, pero el problema de las dimensiones las sitúa en un contexto más complejo en el que las jerarquías de escala comienzan por los principios de prospectiva, prevención y prudencia, a la hora de afrontar una u otra, o varias intervenciones a la vez. Esta es la clave de establecer el reciclaje en la época de la recesión. No por elegir alternativas globales eliminamos incertidumbre, impactos o daños sobrevenidos a la acción principal.

Establecer con sumo cuidado el marco de las dimensiones de intervención no es un trabajo arquitectónico o urbanístico solo. Es un trabajo multi-dimensional, en el que hay que contar con muchos factores, entre ellos los expuestos aquí, pero muchos más si tenemos en cuenta que la materia de la innovación social son las personas y estas no se pueden encasillar en un programa técnico sin más como se ha venido haciendo en muchos casos.

Ninguna duda de que la convivencia de soluciones será larga y no tendrá un sentido unidireccional. La forzada relación de estrategias de progreso con estrategias de involución latente o explícita va a ser la tónica del período que empieza en el $2^{\circ}$ trimestre de 2012, cuando se tengan más claras las dimensiones del ajuste español a las políticas impuestas por Merkozy. Si en los dos últimos años las expectativas de Fondos FEIL, el Plan E y otros permitían afrontar con cierta garantía las políticas keynesianas de crecimiento ahora esas cuestiones están en peligro, incluso en planes de vivienda, en pactos locales y en programas ya aprobados.

Los modos de afrontar determinadas cuestiones no vendrán dados tanto por el inicio de ciclos de vida en barrios, sino por las adaptaciones a escenarios de decrecimiento, a estanflación en recesión o a una precariedad de medios, en la que nuevamente tendrá que volverse a decisiones críticas sobre los mejores modos y modelos de combinar las necesidades sociales con el esfuerzo inversor con las redes existentes o por crear, en el sentido en que pueden ser más 
vitales para el reciclaje las instalaciones de redes Wi-Fi, de salud, de educación, o de intercambio comercial, por poner algunos ejemplos, que las de acción sobre el tejido construido y hay que ser audaces a la hora de elegir cuáles son prioritarias y a cuáles contribuir desde la arquitectura o desde otros ámbitos.

Como ha ocurrido en Málaga, la regeneración de barrios se ha percibido a destiempo, a pesar del esfuerzo en la construcción residencial -en algunos casos especialmente significativosdespués de que se acabaran las infraestructuras de urbanización (Trinidad-Perchel) o de urbanización (calle la Unión y su entorno) o del mercado (Mercado del Carmen en el Perchel) porque hasta entonces la percepción de territorio marginal no se podía suplir con la arquitectura únicamente, por muy digna que esta fuera.

\section{Construir, de-construir, destruir, inventar, innovar, inducir}

Esta aceleración de procesos que ha llevado hasta hoy también se ha puesto de manifiesto que, bajo un acuerdo general de que es mejor rehabilitar, la práctica habitual vuelve a la inercia de construir de nuevo. Si la rehabilitación pasa por la restauración de la comunidad es porque la materia del reciclaje son las personas. Con comunidades marginadas, destruidas o erradicadas es imposible renovar o inventar un territorio, por mucho que esté basado en la eficiencia energética, las energías renovables o instalaciones modernizadas eficientes y seguras. Tampoco cabe imaginar otra manera de reciclar el Hotel Algarrobico en Almería que demolerlo, pero eso es más incierto hoy que nunca.

Las exigencias técnicas del trabajo después del CTE y la progresiva implantación de la Evaluación del Impacto Ambiental EIA de edificios bajo los sistemas actualmente mejor implantadas en nuestro país (GBC, LEED, HV, etc.) no han ido acompañadas de una nueva mirada a la industria de la fabricación y el mantenimiento de la industria más avanzada en estas cuestiones que, a veces, no es la que los arquitectos seleccionamos en primer término en el repertorio del que disponemos. A la cultura de la reutilización le faltan instrumentos de fabricación ecológica en maderas, cales, pinturas, corchos, leds oleds (leds orgánicos), composites y un sinfín de nuevos materiales que están en permanente innovación sin que el sector les de la aplicación masiva que una situación tan extrema desde el punto de vista ambiental y económico ya requeriría. Hablamos de procedimientos, materiales y técnicas que son excelentes porque son responsables; no porque sean o no tradicionales, sino porque son acreditada están homologados, son sostenibles y más baratos de transportar desde lo local y no desde las extravagantes rutas que siguen los materiales de la arquitectura "milagrosa" (Moix, 2010).

Renovar los discursos y la teoría es tan importante como asumir que las materias y los materiales son la base del reciclaje bajo cualquier definición. El uso de materiales ecológicos debe acompañar los esfuerzos para aumentar la sostenibilidad de los microclimas y sistemas urbanos que están en juego. Los agentes de la rehabilitación que incluye reciclaje social no tienen la preparación teórica y práctica en tecnologías, materiales y redes, porque cuando se estaba haciendo la reorientación de esa mano de obra se la expulsó directa y masivamente al desempleo.

\section{5. $\mathbf{R}+\mathbf{R}+\mathbf{R} 2020$}

En 2010 la creación de la Plataforma Social RHÈ+ Fomento de la Rehabilitación, la Accesibilidad y la Eficiencia Energética de Edificios y Viviendas dio lugar al anuncio del pago de ayudas públicas en el plazo de 2 meses desde la finalización de la obra, la colaboración entre el gobierno central y el sector privado con el apoyo 
de una red de Oficinas Técnicas de ayuda al ciudadano, promover la concesión de las licencias de obra menor en un plazo de un mes agilizandolas mediante medios telemáticos, creación de un portal web donde se pueda consultar cualquier duda, ampliación de formación en nuevas tecnologías a trabajadores, agentes, técnicos y empresas. Se hablaba entonces del compromiso de la sociedad con la rehabilitación para un futuro más sostenible a través de un modelo de ciudad en el que primen hogares menos contaminantes y energéticamente más eficientes. El Gobierno impulsaba esta nueva forma de ciudad en línea con los planteamientos de la Estrategia Europa 2020 y con la Agencia Urbana Europea, en la que se apostara por no sólo la sostenibilidad en la edificación desde el punto de vista energético, sino también desde el punto de vista económico. Toda esta inversión implicara de forma sustancial la generación de nuevos puestos de trabajo que ofrecen la regeneración urbana y la rehabilitación aprovechando este potencial económico de un país en que la mitad de los edificios tiene más de 30 años de antigüedad.

Cabe preguntarse qué queda de los buenos proyectos de aquellas plataformas en el momento actual, sin embargo, la estrategia europea 2020 es el único horizonte visible al que podemos mirar para reconducir las prácticas arquitectónicas y urbanísticas de acuerdo con aspiraciones generales más o menos unificadas en nuestro entorno. $Y$ ese es nuestro campo de juego, por mucho que haya que acometer un nuevo esfuerzo de contención presupuestaria, por no decir recortes extraordinarios. Para asumir que los instrumentos como el Plan Concertado de Vivienda y Suelo de Andalucía 2008-2012 pasarán a ser elementos de referencia no hace falta mucha imaginación, dado que la política de vivienda se ha estancado en todas partes por la falta de financiación. La utopía de planes de este perfil parece hoy estar totalmente en contra de las visiones propuestas de Antonio Serrano, entre otros muchos. Parecen descartadas opciones para acometer proyectos como Corviale en Roma, Mario Florentino, 1973-1981, o Toulouse le Mirail en Toulouse Candilis, Josic, Woods, 1962-1972. Hoy las operaciones más efectivas pueden provenir de redes de acciones e iniciativas complementarias, más que de grandes planes integrales que se antojan imposibles, cuando no directamente se vuelven en contra de los proponentes, ante la imposibilidad de que sean finalmente gestionadas.

Reducir será, así pues, el elemento conceptual más seguro, ya que reutilizar, reciclar y rehabilitar son tareas que exigen mucho presupuesto y mucha iniciativa pública, algo que no parece estar en la agenda actual.

Reducir y decrecer en consumo de recursos y suelo solo se puede hacer desde una concepción innovadora que hoy tiene que cambiar las antiguas visiones de los planes y concertarlos con las redes sociales, con nuevas visiones de los arquitectos como innovadores profesionales y con una mayor presencia de los innovadores sociales que están implicados con el espacio que se recicla.

\section{Experiencias, tendencias y cambios} Cabe augurar, -si es que un augurio en esta materia es posible- que tardaremos en encontrar la senda del reciclaje como operación masiva de barrios que puedan reencontrarse con una acción pública de mejora sustancial en instalaciones, ascensores, rehabilitación de viviendas y espacios públicos y que las escalas cuantitativas de las intervenciones serán menores, por ejemplo, que las empleadas en el barrio de San Martín de Porres en Córdoba. Tal vez la tendencia sea a una micronización de 
actuaciones, menos costosas en lo estructural y más participativas en lo social, con implicación efectiva de los afectados en el empleo que se genere o con fórmulas de autogestión en las acciones, -lo que conlleva un cambio de modelo radical respecto al mayoritario seguido hasta ahora-. Las administraciones que han comprometido fondos cuantiosos en los proyectos de regeneración urbana están viendo cómo incumplen sistemáticamente sus promesas.

La tendencia a actuaciones más limitadas a caballo entre el reciclaje y la mejora de servicios y accesos, incluidos aquellos a las energías no contaminantes, a las TICs y a las mejoras del paisaje , la estética urbana y la vegetación serán las consideradas prioritarias ante la falta de financiación, lo que obligará a un extremado acuerdo de cuidado a los vecinos afectados. Lo que quiere decir a acuerdos de participación innovadora con colectivos profesionales y vecinales integrados como los que se mueven en torno a algunas iniciativas como La Casa Invisible o el Mercado de las Artes en Málaga, que buscan soluciones creativas a inmuebles o instalaciones.

Una vuelta a la economía de "trueque" de "concesión por mantenimiento" puede afectar a la gestión de centros sociales o equipamientos, lo que conllevaría a pervertir la noción actual de espacio público por una nueva de espacio colectivo si es que esto es admisible como tal.

Pero tampoco se pueden olvidar las operaciones a mayor escala porque ¿qué otra cosa es el Paseo Marítimo de Benidorm (distinguido también en la BEAU XI) sino una operación a escala de ciudad y paisaje litoral para reciclar de golpe la imagen urbana, la conectividad y los servicios urbanísticos de un vínculo que enlaza el frente urbano en un punto de máxima sensibilidad mediante un alto nivel arquitectónico y estético.

Las tendencias más probables serán a la actuación en forma de marcos de actuación concertada con participación público privada y de los colectivos afectados o las comunidades implicadas, trabajando sobre varios programas a distintas escalas y en red con otros actores locales dentro y fuera de los barrios y los distritos. Se podría decir que la escala micro será una escala red y con más capas y dimensiones de actuación de las que se contemplaban antes en las iniciativas predominantemente constructivas de rehabilitación y de reurbanización. Los planes que puedan realizarse desde una perspectiva holística y con la concentración de varias administraciones serán más del tipo Urban (o FEDER hasta 2013) siguiendo delimitaciones controladas ya tendiendo a problemas muy específicos.

\section{Actores, agentes, emprendedores e innovadores sociales}

Los agentes han cambiado, los actores son otros y los emprendedores de la Gran Recesión son de perfil distinto a los del auge del despilfarro: los modelos cooperativos y colaborativos van a tener nuevos protagonistas y estamos ante un acontecimiento que modificará también los procesos y los procedimientos hasta extremos que ahora no son concebibles. La gestión del nuevo espacio público, en la línea que plantea Daniel Innerarity (2006) tiene que partir de ampliar la noción de acceso, de espacio público y de redes espaciales para nuevos sujetos urbanos más informados, comprometidos, solidarios y efectivos que los que han protagonizado el momento previo a la crisis.

Basta una mirada crítica a los nombres componentes y protagonistas reales de la innovación en materia de ciudades para comprender que nos vamos a encontrar con un escenario distinto, desde A Cidade dos Barrios, Zuloark (Jaque, 2009), Recetas Urbanas, Laboratorio Metropolitano de Madrid, Ecosistema Urbano, Grupo Salvaje CC60, Basurama, Los del Desierto, umdo, On Vas Barcelona, CoRre 2010-2011, 
PKMN, Zira 02, Zoohaus, Casa Leganitos, Motocross, MMMM, Ludotek, etc., y tantos otros colectivos multidisciplinares, se está ofreciendo una alternativa diferente a la planificación centralizada por las administraciones y protagonizada por técnicos del aparato administrativo o liberal.

Eso supone enfrentar el problema desde las propias bases culturales y profesionales de los jóvenes arquitectos, aquellos que han ido dando avisos de la transformación anunciada mucho antes por movimientos y colectivos sociales, finalmente eclosionada por la crisis y difundida por el uso masivo de las redes sociales. Igual ocurre con los grupos universitarios de investigación y con los colectivos sociales reagrupados bajo nuevas bases. Sus interlocutores exceden con mucho los marcos universitarios de investigación, porque sus experiencias son contrastables en el mismo momento en que se están produciendo y se pueden incorporar también como parte activa del proceso de planificación y ejecución.

En otro orden de cosas, emprendedores y empresas también han cambiado los roles y tienen mucho que aportar en solucione suburbanas inteligentes con base en el compromiso social y ambiental. El aprendizaje acerca del valor añadido de la sostenibilidad ha creado poderosos departamentos técnicos en las empresas que comparten muchos planteamientos sobre la necesidad de rehabilitación generalizada, tal como se ha venido demostrando en distintas actuaciones y también en foros ambientales, desde CONAMA a CIBARQ, en proyectos como GBC y en las actuaciones sobre eco-barrios y áreas de rehabilitación preferentes. Claro que la rehabilitación siempre quedó por detrás de la obra nueva dentro de los objetivos del sector en su conjunto.

Esto no es un solar: esta es la cuestión. España se ha convertido en un solar a medio construir y no es un solar, ni debería haberlo sido nunca: nos hemos dejado arrebatar el principal recurso, el de la congruencia de la vida y el barrio. Restaurar una evolución que se ha roto en la dirección opuesta a la esperada va a ser tarea de una nueva generación de actores que vuelvan sobre los pasos de una tradición interrumpida en el camino de la sostenibilidad. Claro que ya en Copenhague 2009, Cancún 2010 y luego en Durban 2011 se sentaron las bases de la involución global en el discurso de la preservación del planeta. En España en 2012 se produce el ataque contra las energías renovables en el escenario más difícil para una revisión del modelo que parecía consolidarse hasta bien entrado 2010, como se viene describiendo hasta ahora.

\section{Reciclaje en Andalucía}

Por lo que se ve en los medios de comunicación la sombra de una vuelta al ladrillo y al chiringuito en nuestra Comunidad Autónoma es más que una amenaza. Parece tratarse de un programa electoral ordenado y meditado hace tiempo por los que creen que la "economía sostenible es una milonga", siempre en la idea de que tenemos que volver la economía tradicional, aunque nos haya llevado a la ruina.

Andalucía ha tenido los mejores instrumentos, metodologías y ejemplos para establecer unas prácticas de autoconstrucción, rehabilitación y restauración en entornos monumentales de toda España. "la Ciudad Viva" ha dado un salto en el que otros territorios autónomos no han tenido tanto acierto.

El problema es que atravesamos momentos de regresión y las prácticas de rehabilitación están siendo acosadas por el discurso del desarrollismo, el reciclaje de barrios por la cultura tradicional y el control de las urbanizaciones ilegales y del litoral pueden sufrir un considerable retroceso si avanza la cultura del "todo vale por el 
empleo" que hay quien sostiene contra viento y marea. Andalucía ha sido el campo de experimentación de las políticas integrales: Un cambio de orientación al escenario actual de ahorro sería el idóneo para vincular más aún los programas a las comunidades y a las redes sociales, a la cultura del territorio sostenible y a la aportación colectiva de los nuevos equipos técnicos puestos al día tanto como los políticos. Esa sería una salida responsable y no un paso atrás irreversible hacia el urbanismo "levantino" que tantas desgracias ha traído sobre la economía de algunas CC.AA.

La reorientación de las políticas públicas a un "urbanismo del cuidado" y la consolidación de nuevos modelos de gestión de la cercanía, de la red y de los proyectos en red, anclados en los sitios y expandidos en la red: Ese sería un camino posible, que hay que hacer, ejemplificar, modelizar y llevar a la práctica, sin acudir a las mismas rutinas que ahora se muestran imposibles de ser realizadas en un largo período. Volcarse en la materia social y en los materiales de bioconstrucción, en las tecnologías de ahorro y las tecnologías sostenibles, reducir, compactar y asumir un arte de la economía de medios, de la estética y de la belleza del paisaje de los barrios como canal hacia la emancipación de las personas.

\section{Estrategias de excelencia en el reciclaje de barrios, distritos y ciudades. Conclusiones}

Las variables de este conjunto de reflexiones conducen a una conclusión general. Tenemos la obligación, como arquitectos, como profesionales comprometidos con nuestro entorno, de seguir avanzando en la senda de la reducción de consumos, de la reutilización de todo el patrimonio edificado que sea susceptible de cambio de uso y a la vez nos toca inventar un cambio de modelo de ahorro nunca conocido hasta ahora, en unas condiciones en que las opciones a elegir serán limitadas si seguimos la vía de la construcción sostenible y serán suicidas si apostamos por las del desarrollo masivo.

Ya no valen las medidas paliativas si queremos servir a la calidad de vida de la gente. Ya no valen mastodónticos programas integrales en todas partes. Hay que poner el zoom en lo local y afianzar las razones que hacen comprometerse a la gente con el hábitat que vive, en el que sueña, disfruta y trabaja.

Los arquitectos debemos estar a la cabeza y en los equipos teóricos y gestores de las transformaciones que vayan en el sentido de dotar de capacidad, aptitudes y competencias a la ciudadanía para que asuma un cambio cultural de proporciones desconocidas en nuestro país. La creatividad y la excelencia de las soluciones urbanas de reciclaje son gran parte de las estrategias de máxima innovación y mucha energía regeneradora. No puede venir impuesta por el nicho de negocio de las multinacionales de las TICs, las energías o las comunicaciones, por las grandes operadoras de los medios internacionales y las grandes operaciones de lavado de cara de las ciudades duales y la investigación, desarrollo e innovación sectorizada, de aquello que podíamos llamar la "domótica de la desigualdad", porque esa será injusta, además de ineficiente.

La escala debe dar la bienvenida a la dimensión y las dimensiones deben abrirse a los flujos de las redes en unos contextos cambiantes en los que el reciclaje de las calidades de vida y de los entornos vendrá a la vez de los cambios y de las aspiraciones a vivir la innovación de los que proyectan y los que construyen el ámbito colectivo del espacio público y alcanzan el desarrollo personal de cada uno en sus ámbitos privados de emancipación libertad. 


\section{Referencias}

BACHELARD, G. La Poética del espacio. México DF: FCE, 1975.

ESTEFANÍA, J. La economía del miedo. Barcelona: Galaxia Gutenberg, 2011.

GLAESER, E. L. El triunfo de las ciudades. Madrid: Taurus, 2011.

GLAESER, E. L. Reinventing Boston: 1640-2003. Discussion Paper Number (Harvard Institute of Economic Research), 2003, № 2017.

GLAESER, E.L., KOLKO, J.; SAIZ, A. Consumer City, Journal of Economic Geography, 2001, no 1, p. 27-50.

GLAESER, E.L., SCHEINKMAN, J.; SHLEIFER, A. Economic Growth in a Cross-Section of Cities, Journal of Monetary Economics, 1995, no 36, p. 117-143.

GLAESER, E.L.; GYOURKO, J. The Impact of Zoning on Housing Affordability, Discussion Paper (Harvard Institute of Economic Research), 2002, ํㅜ 1948.

GLAESER, E.L.; GYOURKO, J. Urban Decline and Durable Housing, NBERWorking Paper, 2001, no 8598

GLAESER, E.L.; KOHLHASE, J.E. Cities, Regions and the Decline of Transport Costs, $\mathrm{Pa}$ pers in Regional Science, 2003.

GÓMEZ NÁRVAEZ, A. Socialistas para un Urbanismo del cuidado. Málaga. 2012.

HERNÁNDEZ PEZZI, C. Ciudad, territorio y talento, Diario Sur, 2011.

HERNÁNDEZ PEZZI, C. La visión de género y el espacio inteligente, Revista feminismos, Alicante, 2011, no 17.

INNERARITY, D. El nuevo espacio público. Madrid: Espasa Calpe SA. HOY, 2006.

JAQUE, A. El arquitecto no es un creador solitario. El País, 2009.

MOIX, L. Arquitectura milagrosa. Hazañas de los arquitectos estrella en la España del Guggen heim. Barcelona: Anagrama, 2010.

PALLASMAA, J. Los ojos de la piel. Barcelona: Editorial Gustavo Gili, 2006.

VEGARA, A.; DE LAS RIVAS, J. L. Territorios Inteligentes. Madrid: Fundación Metrópoli, 2004.

VV.AA. Análisis de las Ciudades Inteligentes en España. IDC Analyze the future. White paper. BBVA. Ferrovial Servicios, IBM, Microsoft, Sage, Telefónica y Urbiótica, 2011.

\section{Cita del artículo}

HERNÁNDEZ PEZZI, C. La excelencia de las transformaciones urbanas a través del reciclaje Hábitat y Sociedad, 2012, ํㅡ 5, p. 11-21

. <www.habitatysociedad.us.es $>$.

http://dx.doi.org/10.12795/HabitatySociedad.2012.i5.02 